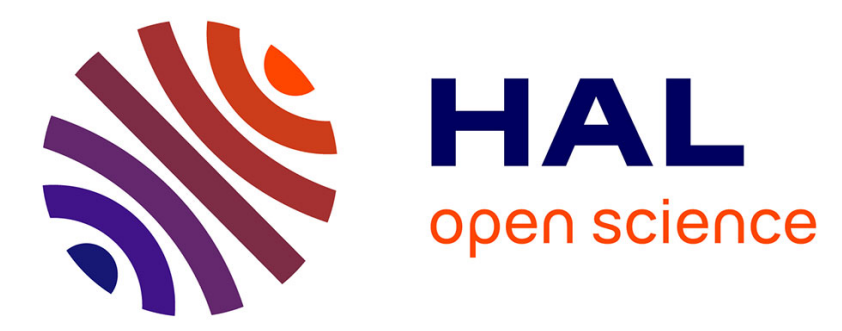

\title{
A Mathematical Morphology Approach to Cell Shape Analysis
}

Jesus Angulo

\section{To cite this version:}

Jesus Angulo. A Mathematical Morphology Approach to Cell Shape Analysis. Luis L. Bonilla, Miguel Moscoso, Gloria Platero, and Jose M. Vega. Progress in Industrial Mathematics at ECMI 2006, part II, Springer, pp.543-547, 2008, Mathematics in Industry, Volume 12, 978-3-540-71991-5. 10.1007/9783-540-71992-2_87. hal-00833838

\section{HAL Id: hal-00833838 \\ https: / hal-mines-paristech.archives-ouvertes.fr/hal-00833838}

Submitted on 13 Jun 2013

HAL is a multi-disciplinary open access archive for the deposit and dissemination of scientific research documents, whether they are published or not. The documents may come from teaching and research institutions in France or abroad, or from public or private research centers.
L'archive ouverte pluridisciplinaire HAL, est destinée au dépôt et à la diffusion de documents scientifiques de niveau recherche, publiés ou non, émanant des établissements d'enseignement et de recherche français ou étrangers, des laboratoires publics ou privés. 


\title{
A mathematical morphology approach to cell shape analysis
}

\author{
Jesús Angulo
}

Centre de Morphologie Mathématique - Ecole des Mines de Paris 35, rue Saint-Honoré, 77300 Fontainebleau, FRANCE jesus.angulo@ensmp.fr; http://cmm.ensmp.fr/ angulo

\section{Introduction: Context and Motivation}

Morphological analysis of cells (size, shape, texture, etc.) is fundamental in quantitative cytology. Anomalies and variations from the typical cell are associated with pathological situations, e.g., useful in cancer diagnosis, in cellbased screening of new active molecules, etc.

Mathematical morphology is a nonlinear image processing technique based on minimum and maximum operations [SER82], i.e., the basic structure is a complete lattice [HEI94]. This contribution aims to apply mathematical morphology operators to quantify the shape of round-objects which present irregularities from an ideal circular pattern. More specifically we illustrate, on the one hand, the application of morphological granulometries for size/shape multi-scale description and on the other hand, the radial/angular decompositions using skeletons in polar-logarithmic representation. We discuss also the aspects related to the properties of invariance of these tools, which is important to describe cell shapes acquired under different magnifications, orientations, etc.

The performance of these mathematical shape descriptors is shown by means of examples from haematological cytology [ANG06] (to classify red blood cells) and from cell-based high-content screening assays [LEM06] (to quantify the populations of hepatocytes), see Fig. 1.
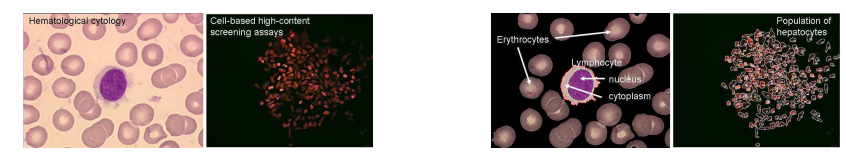

Fig. 1. Microscopic cell images (left), segmented cell shapes to be analysed (right).

Let $E, \mathcal{T}$ be nonempty sets. We denote by $\mathcal{F}(E, \mathcal{T})$ the power set $\mathcal{T}^{E}$, i.e., the set of functions from $E$ onto $\mathcal{T}$. Typically for the digital 2-D images 
$E \subset \mathbb{Z}^{2}$. Let $f$ be a grey level image, $f(x) \in \mathcal{F}(E, \mathcal{T})(x \in E$ is the pixel position), in the case of discrete image values $\mathcal{T}=\left\{t_{\text {min }}, t_{\text {min }}+1, \cdots, t_{\text {max }}\right\}$ (in general $\mathcal{T} \subset \mathbb{Z}$ or $\mathbb{R}$, or any compact subset of $\mathbb{Z}$ or $\mathbb{R}$ ) is an ordered set of grey-levels. We suppose here that a binary image $f$ (sometimes denoted $X$ ) is a two-levels image, i.e., $t_{\min }=0$ and $t_{\max }=1$.

\section{Multi-scale shape descriptors using granulometries}

Given a grey level image $f \in \mathcal{F}(E, \mathcal{T})$, the two basic morphological operators are dilation: $\delta_{n B}(f(x))=\left\{f(y): f(y)=\sup [f(z)], z \in n\left(B_{x}\right)\right\}$, and erosion: $\varepsilon_{n B}(f(x))=\left\{f(y): f(y)=\inf [f(z)], z \in n\left(B_{x}\right)\right\}$, where $B$ is a subset of $\mathbf{Z}^{2}$ and $n \in \mathbf{N}$ a scaling factor. $n\left(B_{x}\right)$ is called structuring element (shape probe) $B$ of size $n$ (homotetic of factor $n$ ) centered at point $x$. Here we suppose that $B$ is plane, symmetric and compact convex. Typically, $n B$ are families of disks (isotropic) or of segments (orientated). Note that $\delta(f), \varepsilon(f) \in \mathcal{F}(E, \mathcal{T})$. Erosion shrinks positive peaks. Peaks thinner than the structuring element disappear. As well, it expands the valleys and the sinks. Dilation produces the dual effects.

The two elementary operations of erosion and dilation can be composed together to yield a new set of operators having desirable feature extractor properties which are opening: $\gamma_{n B}(f)=\delta_{n B}\left(\varepsilon_{n B}(f)\right)$, and closing: $\varphi_{n B}(f)=\varepsilon_{n B}\left(\delta_{n B}(f)\right)$. Opening (closing) removes positive (negative) structures according to the predefined size and shape criterion of the structuring element (smooth in a nonlinear way).
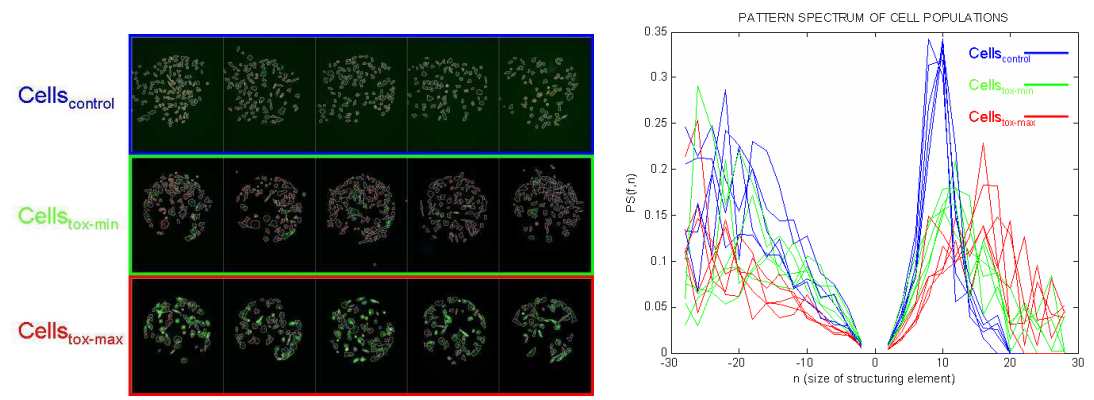

Fig. 2. Left, cell population based high content toxicity biosensor, three examples of toxic concentration. Right, pattern spectra, $P S(f, n)$, with openings (for size/shape description) and closing (for aggregation study) of size $n=-30$ to 30 .

A granulometry is a size distribution based on a pyramid of morphological operators. Formally, it can be defined as an one-parameter family of openings [MAT67] $\Gamma=\left(\gamma_{\lambda}\right)_{\lambda \geq 0}$ such that: (1) $\gamma_{0}$ is the identity mapping, i.e., $\gamma_{0}(f)=f ;(2) \gamma_{\lambda}$ is increasing, i.e., $f \leq g \Rightarrow \gamma_{\lambda}(f) \leq \gamma_{\lambda}(g), \forall \lambda \geq 0, \forall f, g ;(3)$ 
$\gamma_{\lambda}$ is anti-extensive, i.e., $\gamma_{\lambda}(f) \leq f, \forall \lambda \geq 0, \forall f ;(4) \gamma_{\lambda}$ follows the absorption law, i.e., $\forall \lambda \geq 0, \forall \mu \geq 0, \gamma_{\lambda} \gamma_{\mu}=\gamma_{\mu} \gamma_{\lambda}=\gamma_{\max (\lambda, \mu)}$. Moreover, granulometries by closings (or anti-granulometry) can also be defined as families of increasing closings $\Phi=\left(\varphi_{\lambda}\right)_{\lambda \geq 0}$.

The morphological openings, $\gamma_{n B}, B$ compact convex, satisfies the four granulometric postulates. They also satisfy two fundamental properties: (5) the $\gamma_{n B}$ are translation invariant; (6) $\gamma_{n}(f)=\gamma_{1}\left(\frac{1}{n} f\right)$, i.e. there is a unit sieve $\gamma_{1}$, and any other sieve in the process can be evaluated by first scaling the image by the reciprocal of the parameter, filtering by the unit sieve, and then rescaling.

Let $m(f)$ be the Lebesge measure of a discrete image $f$. Performing the granulometric analysis is equivalent to mapping each opening of size $\lambda$ with a measure of the opened image $\gamma_{\lambda}(f)$. The granulometry curve or pattern spectrum [MAR89] of $f$ with respect to $\Gamma$ is defined as the following (normalised) mapping: $P S_{\Gamma}(f, n)=P S(f, n)=\frac{m\left(\gamma_{n}(f)\right)-m\left(\gamma_{n+1}(f)\right)}{m(f)}, n \geq 0$. $P S_{\Gamma}(f)$ maps each size $n$ to some measure of the bright image structures with this size: loss of bright image structures between two successive openings. $P S_{\Gamma}(f)$ is a probability density function (a histogram): a large impulse in the pattern spectrum at a given scale indicates the presence of many image structures at that scale. By duality, the concept of pattern spectra extends to anti-granulometry curve $P S_{\Phi}(f)$ by closings, $P S_{\Phi}(f,-n)=P S(f,-n)=$ $\left(m\left(\varphi_{n}(f)\right)-m\left(\varphi_{n-1}(f)\right)\right) / m(f)$, and is used to characterise the size of dark image structures. The pattern spectrum can be directly used to compare shapes. Moreover different parameters (moments, partial sums, etc.) can be derived from the pattern spectrum to measure the complexity, dispersion, etc. of the shape [SIV97] [BAT97].

Granulometric analysis is very useful to describe the shape of individual cells (see for instance in [ANG06] the cytoplasmic profile classification using the partial sums of $\left.P S_{\Gamma}(f, n)\right)$. Fig. 2 shows an example of application of granulometric analysis to characterise three classes of cell populations (control and two values of toxicity). In this case, the pattern spectra $P S(f, n)$ of segmented cells allow us to classify the populations according to the size/shape of cells (with the family of openings) or with respect to their aggregation (closings).

\section{Radial/angular decompositions using skeletons in log-polar coordinates}

It is difficult to take advantage of radial/angular properties of round-objects (definition of neighbourhood, adapted structuring elements, etc.) when mathematical morphology operators are defined in $\mathcal{F}(E, \mathcal{T})$ (space $E$ corresponds to Cartesian coordinates). The conversion into logarithmic polar coordinates as well as the derived cyclic morphology, recently studied by [LUE04], appears to be a way that provides interesting results to obtain inclusion (extrusion) de- 
compositions by means of angular/radial closings (openings) and to describe shape angularities by computing radial skeletons.

The log-polar transformation converts the cartesian image function $f(x, y)$ : $E \rightarrow \mathcal{T}$ into another $\log$-polar image function $f^{\circ}\left(\rho^{\log }, \theta\right): E_{\rho^{\log _{\theta}}} \rightarrow \mathcal{T}$, where the angular coordinates are placed on the vertical axis and the logarithmic radial coordinates are placed on the horizontal one. More precisely, with respect to a central point $\left(x^{c}, y^{c}\right): \rho=\sqrt{\left(x-x^{c}\right)^{2}+\left(y-y^{c}\right)^{2}}, \rho^{\log }=\log (\rho)$, $0 \leq \rho^{\log } \leq R ; \theta=\arctan \left(\frac{y-y^{c}}{x-x^{c}}\right), 0 \leq \theta<2 \pi$. The support is the space $E_{\rho^{\log \theta}}$, $\left(\rho^{\log }, \theta\right) \in\left(\mathbb{Z} \times \mathbb{Z}_{p}\right)$ (discrete period of $p$ pixels equivalent to $2 \pi$ ). A relation is established where the points at the top of the image $(\theta=0)$ are neighbors to the ones an the bottom $(\theta=p-1)$. The choice of $\left(x^{c}, y^{c}\right)$ is relatively critical. We propose to use the maxima of the distance function or ultimate erosion [SER82]. The image $f^{\circ} \in \mathcal{F}\left(E_{\rho^{\log _{\theta}},}, \mathcal{T}\right)$ presents two properties useful for shape analysis: (1) rotations in the cartesian image $f(x, y)$ become vertical cyclic shifts in the transformed $\log$-pol $f^{\circ}\left(\rho^{\log }, \theta\right)$; (2) the changes of size in $f$ become horizontal shifts in $f^{\circ}$.

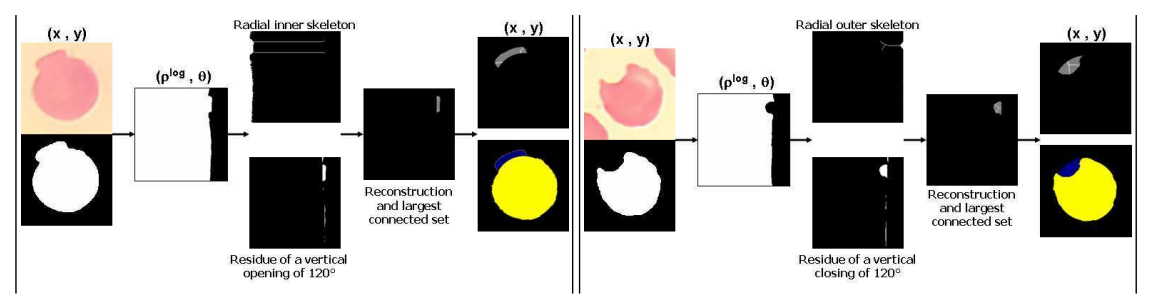

Fig. 3. Erythrocyte shape analysis: morphological algorithm for detecting extrusions (left) and intrusions (right).

The use of classical structuring elements in the log-pol image is equivalent to the use of "radial - angular" structuring elements in the original image, e.g., $g^{\circ}=\delta_{B}\left(f^{\circ}\right)$ where $B$ is a vertical structuring element corresponds in $g$ to the dilation by an arc. (a square in $g^{\circ}$ corresponds to a circular sector in $g$ ). This property yields a method for extracting inclusions/extrusions from the contour of a relatively rounded shape with vertical openings or closings. The proportion of the vertical size from the structuring element with respect to the whole vertical size represents the angle affected in the original cartesian image. With respect to a standard extraction in $E$, the choice of size in $E_{\rho^{\log \theta}}$ is not as critical.

The morphological skeleton by homotopic thinning of a binary image, $\operatorname{skel}(f)$, is a transformation which produces a connected medial axis of the shape [SER82] [HEI92]. However the skeleton of a round object in $E$ is usually biased and for these kind of shapes is more appropriate to work in $E_{\rho^{\log , \theta},}$. Two definitions are possible. The radial inner skeleton skel ${ }_{i n}\left(f^{\circ}\right)$ is the skeleton obtained by an homotopic thinning from the log-pol transformation of an 
object. In the invert transformation to cartesian coordinates, the branches of the radial inner skeleton have radial sense and tend to converge to the center $(\rho=0)$. The radial outer skeleton skel $\mathrm{out}\left(f^{\circ}\right)$ is obtained from the negative image of the log-pol image and in the corresponding cartesian image, the branches tend to diverge to an hypothetical circumference in the infinity $(\rho \longrightarrow \infty)$.

Fig. 3 gives an approach to extract the extrusions/intrusions of red blood cells which is used to classify them according to their shape. The approach for extrusions is composed of several steps: (i) $f \rightarrow f^{\circ}$, (ii) residue of vertical opening $f_{1}^{\circ}=f^{\circ}-\gamma_{B_{\text {vert }}}\left(f^{\circ}\right)$, (iii) radial outer skeleton $f_{2}^{\circ}=\operatorname{skel}_{\text {out }}\left(f^{\circ}\right)$, (iv) reconstruction to extract the connected components associated to the skeleton $f_{3}^{\circ}=\gamma^{r e c}\left(f_{1}^{\circ}, f_{2}^{\circ}\right)$ and $(\mathrm{v}) f_{3}^{\circ} \rightarrow f_{3}$. The algorithm for the intrusions is the same changing the opening by a closing and the radial outer skeleton by a inner skeleton.

\section{References}

[ANG06] Angulo, J., Klossa, J., Flandrin, G.: Ontology-based lymphocyte population description using mathematical morphology on colour blood images. Cellular and Molecular Biology, 52(6), 3-16 (2006)

[LUE04] Luengo-Oroz, M.A., Angulo, J., Flandrin, G., Klossa, J.: Mathematical morphology in polar-logarithmic coordinates. In: Proc. of the 2nd Iberian Conference on Pattern Recognition and Images Analysis (IbPRIA'05), Estoril, Portugal, Springer LNCS 3523, 199-206 (2005)

[HEI92] Heijmans, H.J.A.M.: Mathematical Morphology as a Tool for Shape Description. In: Workshop Shape in Picture, Driebergen, The Netherlands (1992)

[HEI94] Heijmans, H.J.A.M.: Morphological Image Operators. Academic Press, Boston (1994)

[LEM06] Lemaire, F., Mandon, C., Reboud, J., Papine, A., Angulo, J., Pointu, H., Diaz-Latoud, C., Sallette, J., Lajaunie, C., Chatelain, F., Arrigo, A.-P., Schaack, B.: High content toxicity assays in nanodrops reveals individual cell behaviour. PLOS One, (to appear) (2006)

[MAR89] Maragos, P.: Pattern Spectrum and Multiscale Shape Representation. IEEE Transactions on Pattern Analysis and Machine Intelligence, 11(7), 701-716, (1989)

[MAT67] Matheron, G.: Elements pour une théorie des milieux poreux. Masson, Paris (1967)

[SER82] Serra, J.: Image Analysis and Mathematical Morphology, Vol. I., Vol. II Theoretical Advances. Academic Press, London $(1982,1988)$

[SIV97] Sivakumar, K., Goutsias, J.: Discrete morphological size distributions and densities: estimation techniques and application. Journal of Electronic Imaging, 6, 31-53 (1997)

[BAT97] Batman, S., Dougherty, E.R.: Size distributions for multivariate morphological granulometries: Texture classification and statistical propeties. Optical Engineering, 36, 1518-1529 (1997) 